\title{
De l'influence de la communication sur la diffusion artistique
}

Influence of communication upon artistic circulation.

Jean Caune

\section{(2) OpenEdition}

Journals

Édition électronique

URL : http://journals.openedition.org/edc/2795

DOI : $10.4000 /$ edc. 2795

ISSN : 2101-0366

Éditeur

Université Lille-3

Édition imprimée

Date de publication : 1 mai 1991

Pagination : $97-114$

ISSN : $1270-6841$

Référence électronique

Jean Caune, «De l'influence de la communication sur la diffusion artistique », Études de communication [En ligne], 12 | 1991, mis en ligne le 15 janvier 2012, consulté le 01 mai 2019. URL http://journals.openedition.org/edc/2795; DOI : 10.4000/edc.2795

Ce document a été généré automatiquement le 1 mai 2019.

(c) Tous droits réservés 


\section{De l'influence de la communication sur la diffusion artistique}

Influence of communication upon artistic circulation.

Jean Caune

1 Il va sans dire que la communication du produit, ou de l'entreprise, dépend de la nature de l'objet qu'elle promeut, en revanche, l'évaluation des effets de la communication sur là nature du produit, ou l'identité de l'entreprise, est souvent négligée. Le passage de la " réclame » à la publicité transforme une information froide sur l'objet en production d'un désir de l'objet. Mais la modification du processus discursif, qui s'applique d'abord à l'énonciation, a également des effets sur l'énoncé : sur l'objet visé par la communication.

2 L'interrogation sur le débordement fonctionnel du processus de la communication se fait jour, dans l'espace politique, et dans la production d'une opinion publique ${ }^{1}$. Dans le cas de la communication d'un produit artistique (ou d'une institution culturelle), s'est-on suffisamment penché sur l'influence de la stratégie de communication sur la création ou la diffusion artistiques?

3 La communication culturelle n'est pas une simple médiation qui rend manifeste l'existence d'une institution et de ses productions. Elle n'est pas, non plus, le destin d'une information qui solliciterait des réactions réelles ou supposées, symboliques ou désirées du public potentiel. Mise en forme d'un "vouloir-dire » attribué à l'objet artistique, la communication culturelle possède une signification partielle et intentionnelle, destinée à imposer une certaine image du fait artistique, aux yeux d'un destinataire réel, fictif ou imaginaire, pour mieux le séduire, le convaincre ou le persuader. L'opération de communication n'est donc pas un reflet de l'objet artistique. Elle est production d'un rapport social qui anticipe sur le rapport créé par l'objet artistique.

4 Avant même la transaction esthétique - avant que le destinataire ait noué un rapport sensible à l'objet artistique - la communication a déjà orienté et déterminé ce rapport : elle a suscité des attentes. Mais il y a plus, la communication a joué un rôle sélectif : elle a 
opéré un choix, délibéré ou non, dans l'espace public en définissant le public potentiel, ce que les publicitaires appellent la cible.

Si bien que la communication culturelle, en même temps qu'elle est conditionnée, orientée et modelée par les facteurs propres à l'objet communiqué (le produit artistique), est soumise aux circonstances sociales de la diffusion culturelle et à l'idée implicite qu'On se fait du destinataire.

6 Mais qui est ce On, dont la technicité, la légitimité ou encore le pouvoir qui lui est accordé - ou qu'il s'accorde - transforment la médiation en finalité ? L'action de faire connaitre un objet faisant alors s'évanouir l'objet même soumis à la médiation. La communication finit par se suffire à elle-même et conduit à une disparition partielle de l'objet dont l'existence devient secondaire. Combien de spectacles qui ne valent que par la campagne de communication à laquelle ils ont donné lieu ? Combien de représentations qui n'existent que par la juxtaposition de stars sur l'affiche? Combien d'événements culturels qui se réduisent à leur médiatisation?

7 Remarquons en passant que la communication culturelle est la seule qui lance ses produits dans l'espace public sans s'être préalablement préoccupée de son efficacité ou de son impact. Quel meilleur exemple que ces affiches de $4 \mathrm{~m}$ sur $4 \mathrm{~m}$ diffusées sur les panneaux payants de nos villes annonçant un spectacle de théâtre, dans un lieu socialement et culturellement marqué, dont le seul effet est de montrer aux décideurs que la promotion du produit est faite ? Aurait-on l'illusion de penser que les miracles de la publicité feront reculer les ségrégations et les violences symboliques qui s'opposent à la démocratisation culturelle? Mais plutôt que de se formaliser sur j'absence de rentabilité concrète de ces campagnes d'affiches, ne faut-il pas penser qu'ici la communication culturelle a une simple fonction de légitimation? L'objet a de la " valeur » artistique puisqu'il est soumis à une promotion coûteuse.

8 La communication faite autour d'un produit artistique est déjà - avant même la jouissance esthétique, et parfois sans elle - une relation au destinataire.

9 Mais il n'est pas certain que la logique propre à la communication soit accordée à la logique d'usage du produit artistique. De plus, l'émetteur de la communication culturelle (le responsable de la communication) a une certaine idée du destinataire qui n'est pas la même que celle du producteur de l'objet artistique -le sujet réel de l'énonciation artistique. En tout cas, et on peut l'espérer, la relation recherchée dans la transaction esthétique induite par l'objet artistique n'est pas aussi immédiate que celle que réalise la communication.

10 La question est d'autant plus fondamentale que l'objet référentiel de la communication est déjà, en lui-même, une médiation. L'objet d'art, comme le définissait $\mathrm{P}$. Francastel, est un Signe-relais ${ }^{2}$. L'art ne se contente pas d'informer le comportement sensible des individus, il établit des rapports symboliques et imaginaires entre les hommes.

11 Bien plus que forme donnée à la diffusion publique du produit artistique, la communication est stratégie de légitimité de l'institution culturelle, elle est manifestation d'un certain type de rapport social établi avec un certain type de public, enfin elle exprime un point de vue sur l'objet même qu'elle tente d'insérer dans l'espace social.

12 Le processus de communication culturelle - du produit artistique ou de l'institution illustre trois enjeux :

- celui d'une politique culturelle liée à l'art, à sa diffusion, à l'extension du public ; 
- celui du pouvoir de communication propre à l'objet d'art ;

- celui du partage de pouvoir dans l'institution de diffusion et de production.

13 A la fuite du sens, ou à son amputation - risque de mutilation que la communication porte en elle, comme la nuée porte l'orage - se superpose un autre risque : celui que l'objet médiatisé s'adapte aux tropismes et aux exigences de la communication, c'est-à-dire à l'illusion d'une séduction immédiate et éphémère. Mais ce danger n'est-il pas déjà réalisé ?

Dans le domaine de la publicité, des médias audio-visuels et de la fabrication de l'opinion nous en connaissons les effets : banalisation des produits, immédiateté de la signification, appauvrissement de l'implication imaginaire du destinataire, impacts éphémères et fugitifs...

Notre réflexion se propose d'évaluer les risques de contamination de la politique artistique et culturelle par le phénomène de communication. Notre propos se fonde sur l'idée que ce phénomène ne peut se comprendre en dehors de l'évolution des objectifs donnés à la politique culturelle. Nous tenterons dans une première partie de montrer que la confrontation entre la logique de la production et de la diffusion culturelle, d'une part, et les processus de relation aux publics, d'autre part, n'est pas nouvelle et qu'elle suppose une cohérence des objectifs entre l'acte artistique et sa projection dans son espace de diffusion.

Dans un deuxième temps, nous évoquerons la perte du sens qui guette l'objet artistique lorsque la relation aux publics se transforme en relation publique.

\section{Les trois âges de la communication culturelle.}

\section{$1^{\circ}$ Assembler et unir.}

17 En présentant le texte écrit à l'occasion du lancement du T.N.P., en 1951, intitulé « Petit manifeste de Suresnes ", Jean Vilar place en épigraphe une citation de Charles Guieyesse, le premier secrétaire général des Universités populaires. Dans cette citation, C. Guieyesse oppose la connaissance de ceux, ouvriers et paysans qui « ont déjà reçu l'enseignement supérieur de la vie même ", à celle des instituteurs et professeurs qui sont chargés de mettre en ordre l'enseignement de l'éducation populaire. La citation n'est pas innocente, elle témoigne d'un souci constant chez Vilar : réunir ceux que leur naissance ou leur condition ont tenu éloignés de la culture et ceux qui ont déjà le goût du théâtre.

La quasi-totalité des textes de Vilar consacrés au théâtre populaire, ou à la question du public, conduisent à l'affirmation que le théâtre

ne prend sa signification que lorsqu'il parvient à assembler et à unir ${ }^{3}$.

Assembler et unir constituent les objectifs fondamentaux de Vilar.

Si le théâtre doit rassembler les hommes quelles que soient leurs conditions et leurs cultures, cela implique nécessairement un style artistique.

Assembler : cet impératif relève d'une volonté et d'un projet social. L'organisation du public, son information, la recherche de partenaires diversifiés auront pour mission d'élargir le public au-delà des classes privilégiées et de faire de la salle de spectacle un espace de rassemblement. 
Unir : cette injonction est soumise aux effets du spectacle et dépend du répertoire choisi et de son traitement scénique.

Assembler et unir sont les deux objectifs d'une politique théâtrale qui présente deux faces indissociables : la face de l'animateur de l'institution théâtrale ; la face du régisseur, au sens où Vilar utilisait ce terme, puisqu'il n'appréciait pas beaucoup celui de metteur en scène.

L'expérience de l'organisation susceptible de provoquer, d'accroître et d'associer le public est bien connue. Prise en charge par l'administration du T.N.P. et l'association des amis du Théâtre National Populaire (A.T.N.P.), cette action, avant d'être une organisation du public, était la constitution de relais dans les entreprises, les écoles, les lycées, les associations culturelles. Ce schéma de fonctionnement qu'utilisèrent également les troupes de la décentralisation sera exploité plus tard par les institutions de l'Action culturelle.

Le style scénique clair, lisible par tous, expérimenté à Avignon sera celui de Chaillot. Sur la liaison de ces deux caractères constitutifs du théâtre populaire, Vilar a toujours été d'une clarté totale :

Expérience d'un style, recherche d'une organisation, on n'a jamais pu séparer impunément l'une de l'autre. L'une implique l'autre ${ }^{4}$.

A ce propos, il faut faire deux remarques.

La première concerne l'expression d'une contradiction entre, d'une part, un projet artistique, réalisé dans la mise en scène et, d'autre part, une communication sociale qui utilise le théâtre comme moyen de transformation des relations sociales. En 1953, la crise du T.N.P. relève d'une rupture dans cette corrélation. Les Amis du T.N.P. (les ATNP) se séparent du T.N.P. en se transformant en une nouvelle association, les Amis du Théâtre Populaire (A.T.P.), dont le nom indique bien qu'il ne s'agit plus d'une fusion organique avec l'institution du T.N.P. Pour la nouvelle organisation, les A.T.P., le théâtre n'est plus une fin en soi :

Le théâtre, déclare le nouveau président des A.T.P., est pour nous un moyen de faire oeuvre de citoyens 5 .

Il s'établit une rupture entre les objectifs de la communication culturelle fondée sur la diffusion du spectacle et des finalités sociales qui font de l'organisation des spectateurs un moyen d'action civique.

29 Cette rupture préfigure les conflits internes que l'Action culturelle connaîtra, avec plus d'ampleur, entre les producteurs de la représentation théâtrale et les associations qui voudront élargir à une politique culturelle globale la fonction de rassemblement revendiquée par le théâtre populaire.

$30 \mathrm{Ni}$ en 1953, ni plus tard avec le développement des associations de gestion des équipements culturels, le public organisé n'a pu, ni même voulu, se définir autrement que comme usager.

1 La deuxième remarque est relative à la perte de sens d'une communication culturelle qui oublie la spécificité du produit qu'elle diffuse.

32 En s'interrogeant sur la place et la fonction du théâtre dans le phénomène de diffusion culturelle, Vilar annonce toutes les contradictions qui s'épanouiront quelques années plus tard. 
33 En 1962, un an avant son départ du T.N.P., Vilar, dans une " lettre adressée aux associations populaires ", aborde les objectifs qu'une entreprise de théâtre populaire doit se fixer ${ }^{6}$. en pleine santé, si une des raisons de la perte de sens de l'Action culturelle dans les grands équipements, au tout début des années 80 , ne provient pas de ce même phénomène : les relations publiques se sont banalisées pour ne devenir qu'une technique de vente de cartes, d'abonnements et de places.

\section{$2^{\circ}$ L'action culturelle : l'organisation de la rencontre.} le chantre était Malraux. Pour celui-ci, l'oeuvre d'art opère une révélation et réalise une communion. Si bien que la politique d'intervention culturelle de l'État doit s'appuyer sur la dimension artistique, dans la mesure où « l'art est un des plus puissants ferments de la culture ». Les seuls problèmes résident alors dans les questions de diffusion du patrimoine. Il s'agit donc de :

favoriser la communication et la diffusion de l'oeuvre d'art auprès du plus grand nombre en réduisant les obstacles économiques et psychologiques qui écartaient certaines classes sociales de toute communion avec l'œuvre d'art ${ }^{7}$.

Compte tenu de l'accent mis sur l'obstacle économique et la résistance psychologique à la fréquentation de l'oeuvre d'art, il convient de produire des événements, de favoriser l'occasion qui permette à la grâce de l'oeuvre d'atteindre celui qui a été privé de son commerce : l'Action culturelle comme catalyse de l'épiphanie.

La notion de rencontre est centrale chez Malraux : elle fonde le rapport que l'homme entretient avec l'art. 
42 La rencontre dans ce qu'elle a d'éphémère, dans l'inspiration qu'elle provoque, dans la présence qu'elle rend possible, est à l'origine de la communion avec les formes. La rencontre est ce qui permet le partage de "l'héritage ». Dans la rencontre, le chefd'œuvre prend sa place comme réponse à la question que la mort pose à l'homme.

La démocratisation culturelle représente la formulation politique et sociologique d'une conception esthétique fondée sur l'actualisation des oeuvres du passé, par la mise à la disposition au plus grand nombre. C'est vraisemblablement dans cette identification que réside la principale illusion sur le pouvoir de l'Action culturelle. Pour Malraux, le pouvoir de l'art était tel qu'il suffisait de favoriser sa manifestation. Il s'agit alors d'aménager des réseaux de circulation des oeuvres.

Ce sera toute la mission de l'Action culturelle que de susciter ces rencontres. N'oublions pas que si Malraux a pu parler de « cathédrales » pour qualifier les lieux de la rencontre, c'est moins à la dimension architecturale qu'il faisait allusion qu'au fait qu'ils devaient être les lieux de la communion avec l'art.

En dehors de la diffusion du patrimoine, et d'une sensibilisation à l'art, en particulier par le biais de l'animation, l'Action culturelle n'a guère connu d'autre modalité pour rendre présent le phénomène artistique et lui donner une voie et une expression. Une des raisons de l'échec, partiel, de l'Action culturelle sera de n'avoir pas compris que la communion avec l'art ne pouvait se faire sans prendre la mesure des multiples facteurs qui diffèrent le rendez-vous entre le public et l'oeuvre. Une des limites d'une politique d'Action culturelle, fondée sur la diffusion du patrimoine, résidait dans le fait que la simple circulation des oeuvres et des productions artistiques était loin d'être suffisante pour transformer un destin en conscience.

Les thèmes des résistances sociologiques, des inégalités culturelles, des violences symboliques ne sont jamais pris en considération, tant le pouvoir de rayonnement de l'art semble sans limite.

La démocratisation culturelle est perçue comme la conséquence nécessaire du phénomène de recherche d'un vaste public pour les biens culturels. La démocratisation se présente comme le processus qui fait converger l'élargissement du public et l'extension du phénomène de communication artistique.

48 La recherche d'une large audience ; l'espoir que le contact entre un destinataire social et un ensemble énoncé-énonciation produira un sentiment d'appartenance et d'identité ; la valorisation du phénomène de réception au détriment du phénomène d'expression, font de la diffusion du patrimoine un moyen d'action particulier.

La philosophie esthétique des collaborateurs de Malraux pourrait être qualifiée d'esthétique phatique : la confrontation avec l'oeuvre doit être directe.

De la rencontre peut alors naître une familiarité, un choc, une passion, une autre façon peutêtre d'envisager sa propre condition ${ }^{8}$.

50 Le pouvoir de la culture, et de l'art en particulier, n'est donc plus seulement de révélation d'un autre visage du monde, il peut aller jusqu'à bouleverser l'ordre intime de la personne. La découverte de certaines oeuvres, dans certaines conditions, a pu provoquer chez certains des bouleversements allant jusqu'à la remise en cause du mode de vie, mais il était difficile de fonder une politique de diffusion artistique sur ce type d'attente.

51 Il faut insister sur cette conception de la transaction esthétique fondée sur l'immanence du pouvoir de l'oeuvre. 

l'instrument du contact, l'instrument de la transmission, le lieu de la rencontre et de la confrontation. Ainsi, comme par contagion, le pouvoir de l'oeuvre diffuse d'abord dans l'espace social destiné à l'accueillir, et de proche en proche, la Maison de la culture devient le lieu par excellence de la rencontre : entre la culture et ceux qui veulent y accéder, entre ceux qui délivrent le message et ceux qui le reçoivent, entre les artistes et leur public. Dans cette vision théorique, la Maison de la culture intervient comme support et catalyseur de la communication. diffusion allait montrer l'irréalisme d'une telle attente. Comme le reconnaît le rapport d'un groupe d'experts européens,

Malraux n'avait pas de véritable stratégie de démocratisation de la culture.

La conception "stratégique " des Maisons de la culture, dans leur dimension architecturale, et surtout progressivement dans leur pratique d'animation, ne pouvait se limiter à penser que :

pour convertir les non-pratiquants et les entraîner à la reconnaissance de la valeur des oeuvres d'art, il suffisait de les leur présenter'.

Tant que la participation à la culture s'est identifiée à la jouissance des biens culturels, la fonction de l'Action culturelle a pu se définir comme une modalité instrumentale favorisant l'accès du plus grand nombre aux oeuvres du patrimoine.

Dans cette perspective, les établissements d'Action culturelle ont généralisé les techniques et les approches expérimentées par la décentralisation et le T.N.P. De ce point de vue, l'Action culturelle a représenté l'extension, à d'autres disciplines artistiques que le théâtre, et à partir d'équipements diversifiés, d'une volonté de démocratisation culturelle.

Lobjectif d'élargissement du public a contribué à promouvoir une pratique spécialisée l'animation, qui est devenue un instrument de la diffusion du produit artistique.

ex pratiques de relation avec le public et leur extension a été une nécessité organique pour la décentralisation et l'Action culturelle dans sa phase de naissance.

\section{$3^{\circ}$ L'animation culturelle.}

La crise de 1968 mettra en évidence la coupure entre ceux qui bénéficient de la jouissance du patrimoine et ceux qui en sont exclus.

60 Les objectifs donnés à l'Action culturelle dans les années 70 intégreront les préoccupations relatives à l'existence de ce que Francis Jeanson appelait alors le «nonpublic ", et l'animation culturelle cherchera à fournir les supports pour atteindre les couches sociales étrangères aux pratiques culturelles développées par les institutions culturelles.

61 Il appartiendra alors à l'Action culturelle de former un public conscient, de lui donner les moyens d'être concerné par le travail de création et d'adopter une position critique vis-àvis du produit culturel en particulier, et vis-à-vis du monde en général et enfin de susciter sa participation.

Études de communication, 12 | 1991 
62 Mais ce n'est pas tant au créateur, ou à l'objet de sa création, qu'il revient de réaliser ces tâches, mais à un secteur particulier, celui de l'animation culturelle. Elle doit être l'instrument qui lie la création et le public.

Ce n'est donc pas le processus de production artistique qui doit, en lui-même, rechercher un spectateur ou un récepteur actif. L'Action culturelle est conçue comme instrument au service du produit artistique et comme support d'une communication avec le public.

On voit se développer dans les établissements culturels, dans les années 70, des politiques fondées sur la référence prioritaire accordée à l'oeuvre d'art et à sa diffusion. L'animation est alors l'ensemble des techniques destinées à faciliter la rencontre entre le créateur, l'oeuvre et le public. Ces activités qualifiées d'animation-environnement présentent l'oeuvre ou donnent une information sur le spectacle auquel est invité le public. Entrent dans cette catégorie, les diverses interventions " légères » : exposition-spectacle, conférencespectacle, etc. L'animation, dans cette perspective, est une pédagogie qui n'ose s'affirmer en tant que telle. Définie pour servir l'oeuvre artistique, elle est censée en donner, sinon les clefs, du moins les éléments d'information essentiels. Elle laisse de côté tout ce qui peut être de l'ordre des pratiques du processus artistique.

65 L'animation, dans les équipements culturels, est devenue une fonction nécessaire, mais spécialisée, et elle a focalisé toutes les contradictions de l'Action culturelle. Si on examine le dossier publié entre avril et novembre 1972 par l'Association Technique pour l'Action Culturelle (A.T.A.C.), qui regroupe les responsables des établissements culturels subventionnés par le ministère des Affaires culturelles, on peut remarquer que la philosophie de l'animation telle qu'elle est pratiquée sur le terrain est restée contradictoire et ambiguë.

L'animation est perçue comme une fonction essentielle parce que :

tous les individus ne disposent pas des clefs qui ouvrent les diverses portes de l'univers. C'est l'un des rôles de l'animation de combler les lacunes dont souffrent les catégories les plus défavorisées et qui, conjuguées à un véritable conditionnement historique, constituent l'un des principaux obstacles au commerce culturel ${ }^{10}$.

68 C'est à l'animation qu'il reviendra de résoudre la contradiction fondamentale de l'Action culturelle : faire participer au phénomène culturel le plus grand nombre de citoyens, et notamment les plus défavorisés, alors que leurs situations sociales et économiques ne les ont pas préparés à ces pratiques. Mission d'autant plus difficile que les entreprises d'Action culturelle ne peuvent ni masquer les carences, ni pallier les inégalités que ces situations engendrent.

69 L'animation culturelle s'orientera dans deux directions de travail : la première visera à élaborer une pédagogie de sensibilisation et d'initiation à la perception esthétique pour faciliter l'appropriation de la réalité de l'œuvre ; la seconde cherchera à élargir le public au-delà des couches moyennes, à réduire les inégalités culturelles en s'adressant prioritairement aux couches sociales écartées des pratiques artistiques et culturelles.

70 Ces deux directions, bien que présentant des objectifs communs, supposent des méthodes d'intervention différentes. Elles se sont heurtées, toutes deux, à des contraintes qui dépassent de loin le secteur culturel : absence de moyens, insuffisance de liens institutionnels avec l'école, arrêt d'implantation des équipements culturels.

71 Dans un premier temps, les animateurs ont conçu des produits d'accompagnement du spectacle - en général des montages audiovisuels - fondés sur la présentation du contexte historique et social qui a donné naissance à l'oeuvre, ou sur la biographie du créateur et 
son cheminement artistique. C'est surtout pour accompagner la représentation théâtrale que s'est élaborée cette animation concernant directement l'oeuvre, mais elle a pu, dans certains cas, s'établir autour d'expositions, de concerts ou même d'oeuvres littéraires.

Les interventions utilisant des supports légers et spécialisés présentant un point de vue sur l'oeuvre, les thèmes traités, les circonstances, se heurtaient à de multiples limites.

Tout d'abord, il s'agissait d'un commentaire de sens, et rarement d'un éclaircissement du processus artistique. La sensibilisation au langage artistique s'identifiait au " décodage »; la recherche des significations formelles devenait un décryptage du contenu de l'oeuvre. Le commentaire (le métalangage) de l'oeuvre ne peut ni formaliser ni traduire l'expression artistique. Comme le remarque M. Dufrenne, lorsqu'il s'interroge sur la spécificité de l'art par rapport au langage, le sens de l'objet artistique

ne vient pas s'ajouter à la perception, comme son prolongement ou son commentaire, il est

éprouvé au coeur de la perception ${ }^{11}$.

Cela ne veut pas dire pour autant que ce sens puisse atteindre, par transparence, le sujet, qui reçoit l'oeuvre. Bien au contraire, la saisie de l'expression est relayée par la culture. La sensibilité et l'expérience esthétiques sont socialisées et la disponibilité que réclame l'oeuvre passe par un apprentissage. Les interventions se situaient seulement sur le plan du contenu de l'œuvre ; les lectures qu'elles proposaient considéraient l'art comme une langue dont il fallait posséder le code. Ce type d'animation a vite montré ses limites comme transmission édulcorée et émoussée du pouvoir expressif de l'oeuvre.

La deuxième insuffisance de ce type de support destiné à faciliter la rencontre avec l'oeuvre concerne sa situation de dépendance et de service immédiat du produit de " haute qualité ».

76 Ces supports " mimaient " l'oeuvre et se présentaient sous une forme mineure et dévalorisée. L'animation, en tant que fonction spécialisée, s'est constituée autour d'animateurs chargés d'un secteur artistique déterminé. Ceux-ci n'avaient pas simplement la tâche de proposer la programmation de spectacles ou de manifestations, il leur revenait d'inventer les moyens permettant, à la fois d'intéresser telle couche de public, et de le sensibiliser au produit programmé. Tant que le spectacle et la manifestation semblaient répondre à une demande sociale sous-jacente, tant que l'expression artistique présentait une dimension reconnue, partie prenante des modes expressifs intégrés dans la sensibilité esthétique du public traditionnel, la fonction de médiation ou d'initiation n'était pas nécessaire. Il suffisait d'avoir une politique cohérente d'information et d'organisation du public.

77 La politique des établissements ouverts sur la création contemporaine, l'évolution de la mise en scène dans le traitement des classiques, l'impuissance de l'école à promouvoir une formation artistique, le retranchement du public traditionnel derrière des produits consacrés, sont les facteurs qui ont rendu nécessaire une activité dont la mission était de médiatiser le rapport entre l'oeuvre et les publics.

78 La médiation a pris des formes diverses, elle a conjugué des multiples techniques de communication : représentation suivie de débats, montage audiovisuel et exposition, atelier d'expression et réalisation d'un mini-spectacle, audition des différentes versions d'une oeuvre musicale et confrontation des interprétations... Ces systèmes multi-media se présentaient le plus souvent comme une préparation et une ouverture à l'oeuvre programmée dans l'équipement. Ils avaient l'avantage de pouvoir circuler dans des lieux divers, non conçus pour le spectacle. Ces actes d'animation empruntaient très souvent 
leurs supports aux techniques du spectacle, ils se déterminaient par rapport à des oeuvres dont ils constituaient une propédeutique. Cette fonction d'animation ne prenait son sens que par rapport à une fonction dominante, la diffusion des oeuvres. Réduisant l'art à sa dimension communicative, l'animation ne savait que formuler un discours sur l'objet artistique.

\section{Dérives : communiquer l'art, médiatiser la culture.} comportements et des pratiques du public. Se présentant comme une propédeutique, elle facilite l'usage et l'approfondissement de l'objet d'art pour un public de plus en plus large. L'ère de la « reproductibilité technique » permet « à l'objet reproduit de s'offrir à la vision ou à l'audition dans n'importe quelle circonstance $»^{12}$. De plus, comme le remarque W. Benjamin, la réception des oeuvres d'art se fait avec divers accents, et si la valeur de culte de l'objet d'art devient secondaire, la valeur d'exposition prend une dimension prédominante et accentue encore la composante marchande de l'oeuvre. Le nombre de participants au phénomène d'usage de l'art s'est accru, et le mode de participation s'est transformé. Il appartient alors à l'information culturelle de préparer ce nouveau public et de constituer le marché de ces biens culturels. L'information culturelle s'est diversifiée en fonction des langages artistiques (magazines spécialisés), et des publics (public de masse et public élitaire), cette diversification entre presse spécialisée de masse et presse spécialisée élitaire a contribué à produire un champ de pratiques et de compétences (journaliste, critique, expert... ${ }^{13}$. Ce domaine de l'information qui participe pleinement du secteur artistique donne naissance à un discours qui légitime la création artistique, lui facilite son insertion sociale, lui constitue ses publics. Ce discours critique ou métaartistique est un auxiliaire et non un substitut de la production artistique.

Pourtant les risques de dérives affleurent. L'objet d'art n'est certes pas un produit comme les autres, pourtant la publicité est présente dans ce secteur comme dans tout autre secteur marchand. Cherchant à susciter une demande de la part du public potentiel, les techniques publicitaires exercent une stimulation qui bien souvent ignore la ségrégation culturelle et la complexité de la transaction esthétique. Parce qu'elle par nature immédiate et ponctuelle, la publicité relative à l'objet d'art entre en contradiction avec la logique d'usage de l'objet artistique. Il y a bien entendu une composante informative à toute action publicitaire, et celle-ci devient illusoire lorsque l'information publicitaire croit pouvoir dépasser les circonstances socio-culturelles qui sont à l'origine des inégalités. avec les publics en relations publiques.

Dans le phénomène des relations avec les publics, c'est à l'objet d'art ou à l'interprète qu'il appartient de nouer le rapport ultime : la communication est sensibilisation à l'expression artistique.

Dans le système des relations publiques, la communication vise d'abord à constituer une image destinée aux médiateurs. La relation publique établit un décalage à deux niveaux.

Études de communication, 12 | 1991 
Tout d'abord, elle utilise un support de communication qui a peu de rapport avec l'objet artistique qu'elle doit propager dans l'espace public. Ensuite, elle s'adresse à des destinataires dont la légitimité repose sur leur place dans le système des médias.

$\mathrm{Au}$ temps de la décentralisation dramatique et de l'action culturelle, les relations publiques se définissaient comme relations avec les collectivités (associations, syndicats, établissements scolaires...). Ces relations établissaient une structure de relais. Aujourd'hui, les relations publiques sont devenues un service antérieur à la vente du produit. Ces relations fondent une image, elles produisent une «Idole du marché » ${ }^{14}$.

Le propre de l'Idole est de faire prendre les signes conventionnels et l'apparence pour l'essence des choses. Pour ce qui concerne le marché de l'art, l'Idole du marché est d'autant plus trompeuse que l'objet d'art n'existe que par la relation qu'il crée avec celui qui entre en relation d'usage avec lui. L'Idole du marché substitue à la transaction esthétique une transaction avec une image qui n'est rien d'autre que le reflet qu'en donne le système médiatique.

\section{Le créateur, l'animateur, le communicateur et le tiers absent.}

Au-delà des stratégies et des objectifs, ce qui est en jeu dans cette prépondérance du phénomène de la communication aux dépens du phénomène propre à l'objet d'art, c'est le pouvoir dans l'institution culturelle.

Les années 80 ont vu se développer l'opposition, parfois bien artificielle, entre ceux qui se définissaient comme "créateurs » et les animateurs trop vite qualifiés de pédagogues. Cette confrontation n'était pas celle de la création artistique et de l'Action culturelle : celle-ci ne pouvait à aucun prix - sinon à se dénaturer - se passer de l'expression artistique et de l'oeuvre. Ce qui était en jeu était lié à l'exercice du pouvoir dans l'institution.

Quand R. Planchon lance, en 1967, le slogan « le pouvoir aux créateurs », il s'agit d'une affirmation tactique et stratégique. Tactique, parce que le créateur à la tête d'un établissement culturel aurait plus de poids, vis-à-vis du pouvoir politique qu'un animateur. Stratégique parce que Planchon misait sur les qualités du créateur pour marquer de son empreinte, de son style, le fonctionnement et l'action de l'équipement.

L'expérience tentée par J. Lang, au début des années 80 confiant le pouvoir dans les maisons de la culture aux créateurs, n'a pas fait la preuve de son évidence.

91 L'équipement culturel ne peut se passer ni de la création ni de l'artiste et celui-ci peut très bien en assurer la direction. Mais il s'agit alors de la conduite d'un projet qui ne se réduit pas à l'expression du désir du créateur. Les activités des équipements culturels doivent intégrer les circonstances sociales qui conditionnent le rapport à l'art. Ces circonstances se concrétisent dans les idées, les goûts, les normes, les attentes relatives à l'art, tels qu'ils sont transmis par l'éducation, le milieu familial, l'environnement culturel et les médias. C'est dire combien les éléments d'une politique culturelle dans l'équipement relèvent des phénomènes de communication. Encore faut-il les considérer comme débordants les phénomènes de médiatisation.

92 La compréhension des mécanismes de reproduction des inégalités culturelles, la reconnaissance des pratiques diversifiées qui font appel au processus artistique, 
l'appréhension des facteurs qui régissent la sensibilité et le goût esthétique, sont les éléments constitutifs d'une véritable politique de communication de l'objet artistique.

Si la maitrise du pouvoir passe par la maitrise des images, encore faut-il comprendre que le véritable pouvoir consiste à s'opposer à la violence symbolique qui se concrétise dans l'introjection des valeurs, des normes, de modèles véhiculés par la consommation des signes et qui fait passer ce qui est de l'ordre culturel en ordre naturel.

L'oeuvre artistique ne vaut que par l'usage esthétique qu'elle autorise et qu'elle provoque. Cet usage met en jeu une liberté du destinataire de l'oeuvre. Il est clair que cette part de libre-arbitre - de jeu - qui se noue entre une forme et un individu ne se trouve pas totalement dans la forme elle-même. La transaction esthétique suppose un usager averti, sensibilisé et libre par ailleurs. C'est là tout l'enjeu de la communication culturelle : produire les éléments modificateurs qui peuvent contrebalancer les mécanismes de production des inégalités culturelles. C'est dans la production d'un destinataire éclairé, un public actif et sensible, que se situe l'objectif d'une politique artistique et la communication tient un rôle essentiel dans cette perspective.

Il est une autre fonction qu'il faut signaler. En plus de l'accompagnement de la circulation des oeuvres pour utiliser, dynamiser, exploiter, les phénomènes de sens que l'art engendre, il convient d'établir des relations entre le Prince et l'Artiste.

Le face à face exclusif du créateur et du politique, pour déterminer les axes et les modalités d'une politique artistique serait une faillite du politique et une usurpation du pouvoir du créateur. Il n'entre ni dans la compétence du politique, ni dans sa fonction de définir le « beau » ou de le légitimer. Il lui revient en revanche de rendre possible la confrontation de l'oeuvre et des publics et de laisser des aires de liberté aux expressions et aux langages minoritaires. De même, il n'appartient pas au créateur, en tant que créateur, de fixer les démarches artistiques ou de choisir les artisans d'une politique artistique.

La détermination d'une politique artistique passe aussi par la conquête de nouveaux publics. La conduite d'une telle politique ne peut se faire dans la seule confrontation du Prince et de l'Artiste, ce serait perpétuer un mécénat d'État dont les tropismes sont évidents : survalorisation du spectaculaire et des réalisations de prestige, accentuation des modes et du pouvoir des chapelles, délégation des choix artistiques à des conseillers des Princes...

98 C'est l'espace entre le Prince et l'Artiste que la communication culturelle peut espérer remplir. Il s'agit alors, en réintroduisant le tiers manquant, le public, de maîtriser le sens d'une politique artistique. La décision politique ne vaut plus comme choix artistique, pas plus que la création artistique ne vaut comme «éclaireur » du pouvoir. Le pouvoir et le créateur sont inscrits dans un espace public dans lequel circulent des imaginaires, des oeuvres, des expressions, des sensibilités et des pratiques culturelles éclatées. La fonction de la communication n'est plus de construire une image du prince qui utilise la création de l'artiste, pas plus qu'elle n'est de présenter au prince une image séduisante et valorisante de l'artiste. La communication culturelle peut élargir son ambition: insérer la production et la diffusion artistiques dans l'espace social en réintroduisant le tiers manquant : le public potentiel de l'oeuvre. 


\section{BIBLIOGRAPHIE}

Benjamin, W., (1936), «L'oeuvre d'art à l'ère de sa reproductibilité technique », Essais 2, 1935-1940, Denoël/Gonthier.

Champagne, P., (1990), Faire l'opinion, le nouveau jeu politique, Paris, Les Éditions de Minuit.

Copferman, E., (1969), Le théâtre populaire pourquoi ?; Paris, Maspero, p.65.

Dufrenne, M., (1967), Esthétique et philosophie, T. 1, Paris, Klincksieck, p. 107.

Francastel, P., (1970), Études de sociologie de l'art, Paris, Denoël/Gonthier.

Goubert, G., (1972), A.T.A.C. Information, n 40, p. 14.

Guyot, B., (1980), L'information culturelle, Publications de l'Université des langues et lettres de Grenoble.

Programme européen d'évaluation, (1988), La politique culturelle de la France, La documentation française, Paris, p. 31.

Service des études et recherches du ministère des affaires culturelles, (1970), Aspects de la politique culturelle française, U.N.E.S.C.O., Politiques culturelles : études et documents, Paris, p. 12.

Vilar, J., (1975), Le théâtre service public, Paris, Gallimard, p. 144.

\section{NOTES}

1. Champagne, P. (1990), Faire l'opinion, le nouveau jeu politique, Paris, Les Éditions de Minuit.

2. Francastel, P. (1970), Études de sociologie de l'art, Paris, Denoël/Gonthier.

3. Vilar, J. (1975), Le théâtre service public, Paris, Gallimard, p. 144.

4. Id., p. 242.

5. Copferman, E. (1969), Le théâtre populaire pourquoi ?, Paris, Maspero, p.65.

6. Vilar, J., « Lettre aux associations populaires ", dans Op. cit., pp. 364-374.

7. Service des études et recherches du ministère des affaires culturelles (1970), Aspects de la politique culturelle française, U.N.E.S.C.O., Politiques culturelles : études et documents, Paris, p. 12.

8. Ibid.

9. Programme européen d'évaluation (1988), La politique culturelle de la France, La documentation française, Paris, p. 31.

10. Goubert, G. (directeur de la Maison de la culture de Rennes) (1972), A.T.A.C. Information, $\mathrm{n}^{\circ} 40$, p. 14.

11. Dufrenne, M. (1967), Esthétique et philosophie, T. 1, Paris, Klincksieck, p. 107.

12. Benjamin, W. (1936), "L'oeuvre d'art à l'ère de sa reproductibilité technique ", Essais 2, 1935-1940, Denoël/Gonthier.

13. Cf. Guyot, B. (1980), L'information culturelle, Publications de l'Université des langues et lettres de Grenoble.

14. Francis Bacon, dans le Novum Organum, distingue quatre « idoles » ou illusions : les idoles de la tribu, de la caverne, du marché et du théâtre. Cf. Benjamin, W. (1979), Charles Baudelaire, Petite bibliothéque Payot, p.62. 


\section{RÉSUMÉS}

Selon J.Caune, la communication culturelle oriente, avant même que la transaction esthétique n'ait eu lieu, le rapport sensible qu'aura le spectateur à l'objet esthétique. Il étudie dans cette optique trois âges de la vie culturelle : la période vilarienne du théatre comme communication sociale, la période Malraux où l'on a privilégié la « rencontre » entre l'individu et l'oeuvre d'art, la période de l'après 68 , où s'est développée l'animation (socio)culturelle comme médiation.

Il s'interroge sur les effets actuels de la communication culturelle. Elle lui semble plus réussir à imposer une légitimité pour la production de bien culturels, message utile dans les rapports qu'entretiennent créateurs et politiques. Elle n'a pas réussi, à ses yeux, à trouver un langage préparant la rencontre entre le public et l'oeuvre jouée.

According to J.Caune the publicity about cultural events interferes with the spectator's interest for the cultural product, even before the aesthetic response might have taken place. In this viewpoint he studies three periods in culturallife : the Jean Vilar-period which is the theatre as a social communication, the Malraux period, a magical period of epiphany, which favoured the meeting between a work of art and each individual, and then the post- 68 period when the mediatic aspect of artistic circulation started to develop.

He studies the present effects of cultural advertising. In his opinion it mainly seems to legitimate cultural products by making of them a usefullink between dramatists and politicians. For him, it didn't manage to find the kind of language which can lead to an authentic exchange, an aesthetic response of the public to the dramatist.

\section{INDEX}

Mots-clés : communication culturelle, arts du spectacle, médiation culturelle, public, diffusion de la culture

Keywords : cultural communication, performing arts, cultural mediation, public, dissemination of culture

\section{AUTEUR}

\section{JEAN CAUNE}

Jean Caune, Université de Grenoble Stendhal, Ancien directeur de la Maison de la culture de Chambéry 